\title{
THE PRIVATE PROVISION OF A PUBLIC GOOD IS INDEPENDENT OF THE DISTRIBUTION OF INCOME *
}

\author{
Peter G. WARR \\ Australian National University, Canberra, A.C.T. 2600, Australia \\ Received 30 December 1982
}

\begin{abstract}
When a single public good is provided at positive levels by private individuals, its provision is unaffected by a redistribution of income. This holds regardless of differences in individual preferences and despite differences in marginal propensities to contribute to the public good.
\end{abstract}

\section{Introduction}

The effect of a redistribution of income on the private provision of a public good has been an issue in several areas of applied welfare economics. When a public good is privately provided, the level of its provision will typically be sub-optimal from a welfare standpoint. Although public policy measures capable of redressing this situation exist in principle, they may not be implemented. Examples of this will presumably include countries with poorly developed fiscal systems. In such cases, the sub-optimality in the provision of the public good will remain, but it still seems possible that its provision could be affected indirectly by other policy instruments, or external shocks to the economy, via their effects on the distribution of income. It has been assumed in the literature that these indirect effects will occur because individuals differ in their marginal propensities to contribute to the provision of the public good. ${ }^{1}$

* This paper has benefited from the author's discussions with J.J. Pincus and Brian D. Wright and from the suggestions of a referee.

${ }^{1}$ For a development of this theme in the context of benefit-cost analysis, see Dasgupta, Marglin and Sen (1972). 
To the extent that a redistribution of income affects the provision of an under-supplied public good, welfare will indeed be affected. But this note shows that an interesting class of cases exists in which the level of provision of a public good is independent of the distribution of income. This occurs when individuals behave as atomistic utility maximizers in the determination of their provision of a single public good, and where this results in an interior solution to their utility maximization problem. In such cases a redistribution of income has no effect on the level of provision, regardless of differences in individuals' marginal propensities to contribute.

\section{The model}

We imagine an economy containing $n$ consumers. The utility of each depends on his consumption of each of $m$ private goods and on the aggregate provision of a public good. ${ }^{2}$ Individual $i$ 's consumption of private goods is denoted by the vector $c^{i}=\left(c_{1}^{i}, c_{2}^{i}, \ldots, c_{m}^{i}\right)$, where $c_{k}^{i}$ denotes his consumption of good $k$. The aggregate provision of the public good is denoted $g$. Thus the utility of individual $i$ is written $U^{i}=U^{i}\left(c^{i}, g\right)$. The functions $U^{i}$ are each assumed to be strictly quasi-concave, twice differentiable and increasing in all arguments, but individual utility functions need not be the same.

Each individual receives an income, denoted $y^{i}$, which is determined from outside the model. He then allocates this income between expenditure on each of the $m$ private goods and expenditure on the public good. The prices of the private goods are denoted by the vector $p=\left(p_{1}, p_{2}, \ldots\right.$ $p_{m}$ ), and the price of the public good is denoted by $q$. Individual $i$ 's budget constraint is thus $y^{i} \geqq p \cdot c^{i}+q g^{i}$, where $g^{i}$ and $q g^{i}$ denote this voluntary provision of the public good, and his expenditure on it, respectively. Obviously, $g=\sum_{i=1}^{n} g^{i}$. Each individual behaves as a utility maximizing competitor in the determination of his provision of the public good and his consumption of private goods. Clearly, his budget constraint will be satisfied as a strict equality.

2 This public good therefore corresponds to the 'collective consumption good' described by Samuelson (1954). For useful discussions of Samuelson's concept, see Head (1962), Ng (1979), and Atkinson and Stiglitz (1980). 
This implies the familiar Kuhn-Tucker conditions

$c_{k}^{i}\left(U_{k}^{i}-\lambda p_{k}\right)=0, \quad k=1,2, \ldots, m, \quad$ and

$g^{i}\left(U_{g}^{i}-\lambda q\right)=0$,

where, as usual, $U_{k}^{i}=\partial U^{i} / \partial c_{k}^{i}, \partial U_{x}^{i}=\partial U^{i} / \partial g$ and $\lambda$ is a Lagrangean multiplier. We will focus on the case where the public good is provided at positive levels and (solely for convenience) each of the private goods is consumed at positive levels. This implies $m$ equations of the form

$U_{k}^{i} / U_{g}^{i}=p_{k} / q, \quad k=1,2, \ldots, m$.

It is well known, and easily confirmed within this model, that the equilibrium provision of the public good that emerges from (3) is below the level consistent with Paretian optimality. We now examine whether this level of provision could be affected by a redistribution of income.

\section{The basic result}

Each of the equations from (3) can be rewritten $J_{k}^{i}\left(c^{i}, g\right)=p_{k} / q$ and, utilizing the implicit function theorem, these $m$ equations can be solved to give

$c_{k}^{i}=H_{k}^{i}(p, q, g), \quad k=1,2, \ldots, m$.

From the budget constraint of individual $i$,

$y^{i}-q g^{i}=p \cdot c^{i}=\sum_{k=1}^{m} p_{k} H_{k}^{i}(p, q, g)$.

We now sum these equations across the $n$ individuals, writing $y=\sum_{i=1}^{n} y^{i}$ for aggregate income. This gives

$y-q g=\sum_{k=1}^{m} p_{k} \sum_{i=1}^{n} H_{k}^{i}(p, q, g)$.

This equation can now be solved for $g$ to give the aggregate demand 
function

$g=D_{g}(p, q, y)$

The demand for the public good depends on the prices of private goods, the price of the public good, and aggregate income. It does not depend on the distribution of income. But we have reached this conclusion without assuming that individuals have identical tastes. Moreover, the aggregate demand for private good $k$ is, from (4) and (7),

$c_{k}=\sum_{i=1}^{n}, \quad c_{k}^{i}=\sum_{i=1}^{n} H_{k}^{i}\left(p, q, D_{g}(p, q, y)\right)$.

This can be rewritten $c_{k}=D_{k}(p, q, y)$. The aggregate demand for each of the private goods is also independent of the distribution of income. In this model, a pure redistribution of income does not affect the demand for the public good or any private good, and since it obviously does not affect the supply of any good, no price will be affected. Consequently, from (7), the equilibrium provision of the public good is independent of the distribution of income.

From (4), we now see that each individual's consumption of each private good, and hence his expenditure on private goods, is similarly unaffected by a pure redistribution. This implies that if we consider a vector of changes to individual incomes $\left(\mathrm{d} y^{1}, \mathrm{~d} y^{2}, \ldots, \mathrm{d} y^{n}\right)$ such that $\sum_{i=1}^{n} \mathrm{~d} y^{i}=0$, then

$q \mathrm{~d} g^{i}=\mathrm{d} y^{i}, \quad i=1,2, \ldots, n$,

where $\mathrm{d} g^{i}$ is the equilibrium adjustment of individual $i$ 's provision of the public good in response to the redistribution. The effect that a pure redistribution has on each individual is absorbed entirely by adjustments to his expenditure on the public good; but aggregate provision of the public good does not change and individual utilities are unaffected.

\section{Extensions}

The key to the derivation of the basic result is the decomposition by which individual budget equations are aggregated to give an equation system in which aggregate income and aggregate provision of the public 
good appear but individual income levels do not. The original system contains $n m$ first order conditions and $n$ budget equations, giving $n(m+1)$ equations in $n(m+1)$ endogenous variables - $n m$ individual consumption levels for private goods and $n$ levels of individual provision of the public good. Adding the budget equations leaves $n m+1$ equations in $n m+1$ variables; the number of equations deleted in this decomposition $(n-1)$ matches the number of variables that are lost. It is obvious from (3) and (9) that the result requires that each individual who is affected by a redistribution $\left(\mathrm{d} y^{i} \neq 0\right)$ was initially providing the public good in positive quantity, and that $q g^{i} \geqq-\mathrm{d} y^{i}$. But the result is not disturbed if some or all individuals do not consume all $m$ private goods in positive quantity, provided each consumes at least one private good. For each corner solution with respect to private goods, one variable $\left(c_{k}^{i}\right)$ and one equation (first-order condition) are deleted from the system.

The analysis does not extend directly to a model containing two or more separate public goods. Suppose there were $r$ public goods, each provided at positive levels by each individual. There would now be $n(m+r-1)$ first order conditions and $n$ budget equations, a total of $n(m+r)$ equations in $n(m+r)$ endogenous variables. Aggregating budget equations as before causes $n-1$ equations to be deleted, but $r(n-1)$ variables are lost also. In this decomposed system the number of equations exceeds the number of variables by $(r-1)(n-1)$. The analysis applies without amendment only for $r=1$. But if $r>1$ the analysis continues to apply if one of these public goods is privately provided at positive levels by each individual and the other $r-1$ are either not provided at all or are provided collectively. The aggregate provision of these $r-1$ public goods is then exogenous to the relevant individual utility maximization conditions.

\section{References}

Atkinson, A.B. and J.E. Stiglitz, 1980, Lectures on public economics (McGraw-Hill, New York).

Dasgupta, P., S.A. Marglin and A.K. Sen, 1972, Guidelines for project evaluation (United Nations, New York).

Head, J.G., 1962, Public goods and public policy, Public Finance/Finances Publiques 17, 197-219, reprinted in: J.G. Head, 1974. Public goods and public welfare (Duke University Press, Durham, NC) 164-183.

Ng, Y-K., 1979, Welfare economics (Macmillan, London).

Samuelson, P.A., 1954, The pure theory of public expenditure, Review of Economics and Statistics $36,387-389$. 